Homology, Homotopy and Applications, vol.15(1), 2013, pp.223-233

\title{
ON TRIVIALITIES OF STIEFEL-WHITNEY CLASSES OF VECTOR BUNDLES OVER ITERATED SUSPENSIONS OF DOLD MANIFOLDS
}

\author{
AJAY SINGH THAKUR
}

(communicated by Graham Ellis)

\begin{abstract}
A space $X$ is called $W$-trivial if for every vector bundle $\xi$ over $X$, the total Stiefel-Whitney class $W(\xi)=1$. In this article we shall investigate whether the suspensions $\Sigma^{k} D(m, n)$ of Dold manifolds are $W$-trivial or not.
\end{abstract}

\section{Introduction}

Recall [11] that a CW-complex $X$ is said to be $W$-trivial if for any vector bundle $\xi$ over $X$, the total Stiefel-Whitney class $W(\xi)=1$.

It is a theorem of Atiyah-Hirzebruch [1, Theorem 2] that the 9-fold suspension $\Sigma^{9} X$ of any $\mathrm{CW}$-complex $X$ is $W$-trivial (see also [11, Corollary 1.2]). In the same paper, Atiyah-Hirzebruch [1, Theorem 1] have shown that the sphere $S^{d}=\Sigma^{d} S^{0}$ is $W$-trivial if and only if $d \neq 1,2,4$ and 8 (see also [7, Theorem 1]). Here $S^{0}$ is the union to two distinct points.

It is therefore an interesting question to understand for what value of $k, 0 \leqslant k \leqslant 8$, is the iterated suspension $\Sigma^{k} X$, of a CW-complex $X, W$-trivial. Another motivation to study the $W$-triviality of a CW-complex is its connection with $I$-triviality [9]. If a CW-complex $B$ is $W$-trivial, then it is $I$-trivial, and hence it satisfies a BorsukUlam type theorem. We refer to $[\mathbf{9}]$ and $[\mathbf{1 2}]$ for more details on $I$-triviality of a CW-complex.

In [10], R. Tanaka obtained results concerning the $W$-triviality and " $W$-triviality except at one dimension" for highly connected CW-complexes. In [11], R. Tanaka determined all pairs $(k, n)$ of positive integers for which $\Sigma^{k} \mathbb{F} \mathbb{P}^{n}$ is $W$-trivial, where $\mathbb{F}=\mathbb{R}, \mathbb{C}$ or $\mathbb{H}$.

In this article we shall investigate when the iterated suspension $\Sigma^{k} D(m, n)$, of the Dold manifold $D(m, n)$ is $W$-trivial. Recall [2] that the Dold manifold $D(m, n)$ is an $(m+2 n)$-dimensional manifold defined as the quotient of $S^{m} \times \mathbb{C P}^{n}$ by the fixed point free involution $(x, z) \mapsto(-x, \bar{z})$. The projection $S^{m} \times \mathbb{C P}^{n} \longrightarrow S^{m}$ gives rise to a fiber bundle

Most of this work was done when the author was a post-doc at Indian Statistical Institute, Bangalore, India.

Received September 11, 2012, revised November 15, 2012; published on April 30, 2013.

2000 Mathematics Subject Classification: 57R20; 55R40, 57R22.

Key words and phrases: Stiefel-Whitney class, Dold manifold, suspension, $K$-theory.

Article available at http://intlpress.com/HHA/v15/n1/a11 and doi:10.4310/HHA.2013.v15.n1.a11

Copyright (C) 2013, International Press. Permission to copy for private use granted. 


$$
\mathbb{C P}^{n} \hookrightarrow D(m, n) \longrightarrow \mathbb{R P}^{m}
$$

with fiber $\mathbb{C}^{n}$ and structure group $\mathbb{Z}_{2}$. In particular, we have $D(m, 0)=\mathbb{R} \mathbb{P}^{m}$ and $D(0, n)=\mathbb{C P}^{n}$.

By the theorem of Atiyah-Hirzebruch [1, Theorem 2], $\Sigma^{k} D(m, n)$ is $W$-trivial for $k \geqslant 9$. So we shall be interested only in the case $0 \leqslant k \leqslant 8$ and $m>0$. We have the following main results:

Theorem 1.1. Let $\Sigma^{k} D(m, n)$ be the $k$-fold suspension of the Dold manifold $D(m, n)$ with $m>0$. Then $\Sigma^{k} D(m, n)$ is not $W$-trivial if

1. $k=0$.

2. $k=1,2,4$ or 8 and $m \geqslant k$.

3. $k=3,5$ or 7 and $m+k=4$ or 8 .

4. $k=6$ and $m=2$ or 3 .

Theorem 1.2. Let $\Sigma^{k} D(m, n)$ be the $k$-fold suspension of the Dold manifold $D(m, n)$ with $m>0$ and $n$ even. Then $\Sigma^{k} D(m, n)$ is $W$-trivial if

1. $k=2$ and $m=1$.

2. $k=3$ and $m \neq 5,8 t+1$.

3. $k=4$ and $m=3$.

4. $k=5$ and $m \neq 8 t+3$.

5. $k=6$ and $m \neq 2,3,8 t+4$.

6. $k=7$ and $m \neq 1,8 t+5$.

7. $k=8$ and $m=1,2,3$ or 7 .

We have the following result in the case when $n$ is odd:

Theorem 1.3. Let $\Sigma^{k} D(m, n)$ be the $k$-fold suspension of the Dold manifold $D(m, n)$ with $m>0$ and $n$ odd. Then $\Sigma^{k} D(m, n)$ is $W$-trivial if $k$ and $m$ are as listed above in (1)-(7) of Theorem 1.2 and any one of the following conditions is satisfied:

1. $n+k=2,4$ or 8 and $m<k$.

2. $n+k=3,5$ or 7 and $2 n+m+k \neq 4,8$.

3. $n+k=6$ and $m+n \neq 2,3$.

4. $n+k \geqslant 9$.

Observe that if we assume $n \geqslant 3$ in Theorem 1.3 , then $\Sigma^{k} D(m, n)$ is $W$-trivial except for $\Sigma^{3} D(m, 5)$ with $m \geqslant 3$ and $\Sigma^{5} D(m, 3)$ with $m \geqslant 5$. In the case when $n=1$, we have the following theorem:

Theorem 1.4. Let $\Sigma^{k} D(m, 1)$ be $k$-fold suspension of the Dold manifold $D(m, 1)$ with $m>0$. Then $\Sigma^{k} D(m, 1)$ is not $W$-trivial if

1. $k=1,3$ or 7 and $m \geqslant k$.

2. $k=2$ or 4 and $m+k=2$ or 6 .

3. $k=5$ and $m=1$ or 2 . 
We shall also prove that, for $n>1$ and $n \not \equiv 3(\bmod 4)$, the 4 -fold suspension $\Sigma^{4} D(1, n)$ is not $W$-trivial.

Following are the cases which we have not been able to settle: (i) $k=3$ and $m=$ $8 t+1$, (ii) $k=4$ and $m=2$, (iii) $k=5$ and $m=8 t+3$, (iv) $k=6$ and $m=8 t+4$, (v) $k=7$ and $m=8 t+5$ and (vi) $k=8$ and $m=4,5$ or 6 . In addition to these cases, we also do not know whether $\Sigma^{k} D(m, n)$ is $W$-trivial or not when $k, m$ and $n$ satisfy any one of the following conditions: (i) $k=3, n=5$ and $m \geqslant 5$, (ii) $k=5, n=3$ and $m \geqslant 7$ and (iii) $k=4, m=1$ and $n \equiv 3(\bmod 4)$.

To prove our results we shall require the description, by Fujii-Yasui [6], of $\mathrm{KO}-$ groups of Dold manifolds. We shall recall this in Section 2 and prove our results in Section 3 .

\section{Acknowledgements}

The author is grateful to Aniruddha Naolekar for several valuable discussions. He also thanks him for his helpful comments and suggestions on the manuscript.

\section{Preliminaries}

In this section we shall recall the notations and results from [6], where M. Fujii and T. Yasui have computed the $K O$-groups of Dold manifolds. These will be used to prove our results.

Let $\pi: D(m, n) \rightarrow D(m, n) / D(m, 0)$ be the projection. Let $q: D(m, n) \rightarrow \mathbb{R P}^{m}$ be the projection map of the fiber bundle, which is described in the introduction, and let $i: D(m, 0) \hookrightarrow D(m, n)$ be the inclusion defined by

$$
i\left(\left[x_{0}, x_{1}, \ldots, x_{m}\right]\right)=\left[x_{0}, x_{1}, \ldots, x_{m}, 1,0, \ldots, 0\right] .
$$

Consider the following exact sequence of $\widetilde{K O}$-groups:

$$
\cdots \rightarrow \widetilde{K O}^{-k}(D(m, n) / D(m, 0)) \stackrel{\pi^{!}}{\rightarrow} \widetilde{K O}^{-k}(D(m, n)) \stackrel{i^{!}}{\rightarrow} \widetilde{K O}^{-k}(D(m, 0)) \rightarrow \cdots .
$$

Under the identification $D(m, 0)=\mathbb{R}^{m}$, we have the composition $i^{!} \circ q^{!}=$identity. Hence the homomorphism

$$
q^{!}: \widetilde{K O}^{-k}\left(\mathbb{R P}^{m}\right)=\widetilde{K O}^{-k}(D(m, 0)) \rightarrow \widetilde{K O}^{-k}(D(m, n))
$$

is an injective map, and it gives the splitting of the exact sequence (1). Let

$$
\widetilde{K O}^{-k}(m, n):=\pi^{!} \widetilde{K O}^{-k}(D(m, n) / D(m, 0)) .
$$

Then we have the following theorem:

Theorem 2.1 ([6, Theorem 1]).

$$
\widetilde{K O}^{-k}(D(m, n))=\widetilde{K O}^{-k}(m, n) \oplus q^{!} \widetilde{K O}^{-k}\left(\mathbb{R P}^{m}\right),
$$

where $q: D(m, n) \rightarrow \mathbb{R P}^{m}$ is the natural projection.

The $K O$-groups of the projective space $\mathbb{R}^{m}$ have been studied by M. Fujii in [3]. The group $\widetilde{K O}^{-k}(m, n)$ has been computed by M. Fujii and T. Yasui in [6] by making 
use of the following two homeomorphisms [4, Proposition 2]:

1. $h_{1}: D(m, n) / D(m-1, n) \approx S^{m} \wedge\left(\mathbb{C P}^{n}\right)^{+}$.

2. $h_{2}: D(m, n) / D(m, n-1) \approx S^{n} \wedge\left(\mathbb{R} \mathbb{P}^{m+n} / \mathbb{R P}^{n-1}\right)$.

Here, for a space $X, X^{+}$denotes the disjoint union of $X$ and a point. The identification of the spaces via homeomorphisms $h_{1}$ gives rise to the following long exact sequence [6, p. 58]:

$$
\begin{aligned}
\cdots \longrightarrow \widetilde{K O}^{-k}\left(S^{m} \wedge \mathbb{C P}^{n}\right) \stackrel{f^{!}}{\rightarrow} \widetilde{K O}^{-k}(m, n) \stackrel{i^{!}}{\rightarrow} \widetilde{K O}^{-k}(m-1, n) \\
\stackrel{\delta}{\rightarrow} \widetilde{K O}^{-i+1}\left(S^{m} \wedge \mathbb{C P}^{n}\right) \longrightarrow \cdots,
\end{aligned}
$$

where $f=h_{1} \circ \pi$ and $i: D(m-1, n) \hookrightarrow D(m, n)$ is the inclusion. The long exact sequence (2) is a direct summand of the following long exact sequence of $\widetilde{K O}$-groups for the pair $(D(m, n), D(m-1, n))$ :

$$
\rightarrow \widetilde{K O}^{-k}(D(m, n) / D(m-1, n)) \stackrel{\pi^{!}}{\rightarrow} \widetilde{K O}^{-k}(D(m, n)) \stackrel{i^{!}}{\rightarrow} \widetilde{K O}^{-k}(D(m-1, n)) \rightarrow \cdots
$$

In the case when $n=2 r$, the groups $\widetilde{K O}^{-k}(m, 2 r)$ have been described in $[\mathbf{6}$, Theorem 3]. The proof of the following lemma follows directly from [6, Theorem 3] by counting the generators of $\widetilde{K O}^{-k}(m, 2 r)$.

Lemma 2.2. Let $\widetilde{K O}^{-k}(m, 2 r):=\pi^{!} \widetilde{K O}^{-k}(D(m, 2 r) / D(m, 0))$, where $\pi$ is the projection. Let $m>0$. Then $\widetilde{K O}^{-k}(m, 2 r)=0$ if

1. $k=2$ and $m=1$.

2. $k=3$ and $m=8 t+2,8 t+3,8 t+4$ or $8 t+6$.

3. $k=5$ and $m=8 t, 8 t+4,8 t+5$ or $8 t+6$.

4. $k=6$ and $m=8 t+1,8 t+5,8 t+6$ or $8 t+7$.

5. $k=7$ and $m=8 t, 8 t+2,8 t+6$ or $8 t+7$.

In the case when $n=2 r+1$, the long exact sequence of $\widetilde{K O}$-groups for the pair $(D(m, 2 r+1), D(m, 2 r))$ takes the following form [6, p. 55]:

$$
\rightarrow \widetilde{K O}^{-k}(D(m, 2 r+1) / D(m, 2 r)) \rightarrow \widetilde{K O}^{-k}(m, 2 r+1) \stackrel{i_{1}^{!}}{\rightarrow} \widetilde{K O}^{-k}(m, 2 r) \rightarrow,
$$

where $i_{1}: D(m, 2 r) \hookrightarrow D(m, 2 r+1)$ is the inclusion. For the long exact sequence (3), there exists an algebraic splitting homomorphism

$$
\kappa: \widetilde{K O}^{-k}(m, 2 r) \rightarrow \widetilde{K O}^{-k}(m, 2 r+1)
$$

such that $i_{1}^{!} \circ \kappa=$ identity (refer to [6, Section 10]). In fact, the homomorphism $\kappa$ is defined as the composition $i_{2}^{!} \circ p$, where $i_{2}: D(m, 2 r+1) \hookrightarrow D(m, 2 r+2)$ is the inclusion and $p: \widetilde{K O}^{-k}(m, 2 r) \rightarrow \widetilde{K O}^{-k}(m, 2 r+2)$ is an algebraic homomorphism defined in [6, Section 10]. Therefore we have the following theorem:

Theorem 2.3 ([6, Theorem 2]).

$$
\widetilde{K O}^{-k}(m, 2 r+1)=\widetilde{K O}^{-k}(m, 2 r) \oplus \widetilde{K O}^{-k}(D(m, 2 r+1) / D(m, 2 r)) .
$$


In the above direct sum decomposition, the group $\widetilde{K O}^{-k}(m, 2 r)$ is a direct summand of $\widetilde{K O}^{-k}(m, 2 r+1)$ via the monomorphism $\kappa$, and the group $\widetilde{K O}^{-k}(D(m, 2 r+$ $1) / D(m, 2 r))$ is isomorphic to $\widetilde{K O}^{-k}\left(S^{2 r+1} \wedge\left(\mathbb{R}^{2 r+1+m} / \mathbb{R}^{2 r}\right)\right)$ by the homeomorphism $h_{2}$. The latter group, $\widetilde{K O}^{-k}\left(S^{2 r+1} \wedge\left(\mathbb{R P}^{2 r+1+m} / \mathbb{R P}^{2 r}\right)\right)$, has been computed by M. Fujii and T. Yasui in [5].

\section{Proof of the main results}

We first state the following well-known facts which we shall use implicitly in our proofs:

1. For a vector bundle $\xi$ over a $\mathrm{CW}$-complex $X$, the smallest integer $k>0$ with $w_{k}(\xi) \neq 0$ is a power of 2 (see, for example, [8, p. 94]).

2. If $\widetilde{K O}(X)=0$ then every vector bundle over $X$ is stably trivial, and hence $W(\xi)=1$ for any vector bundle $\xi$ over $X$. Thus $X$ is $W$-trivial.

3. Recall $[\mathbf{2}]$ that the $\mathbb{Z}_{2}$-cohomology ring of the Dold manifold $D(m, n)$ is given as

$$
H^{k}\left(D(m, n) ; \mathbb{Z}_{2}\right)=\mathbb{Z}_{2}[c, d] /\left(c^{m+1}=0, d^{n+1}=0\right),
$$

where $c \in H^{1}\left(D(m, n) ; \mathbb{Z}_{2}\right)$ and $d \in H^{2}\left(D(m, n) ; \mathbb{Z}_{2}\right)$. If $c^{\prime}$ is the generator of $H^{1}\left(\mathbb{R P} P^{m} ; \mathbb{Z}_{2}\right)$ and $d^{\prime}$ is the generator of $H^{2}\left(\mathbb{C P}^{n} ; \mathbb{Z}_{2}\right)$, then $q^{*}\left(c^{\prime}\right)=c$ and $i^{*}(d)=d^{\prime}$, where $q: D(m, n) \rightarrow \mathbb{R P}^{m}$ is the projection and $i: \mathbb{C P}^{n} \hookrightarrow D(m, n)$ is the fibre inclusion of the fibre bundle

$$
\mathbb{C P}^{n} \stackrel{i}{\hookrightarrow} D(m, n) \stackrel{q}{\longrightarrow} \mathbb{R P}^{m} .
$$

The action of Steenrod squares on the cohomology ring $H^{*}\left(D(m, n) ; \mathbb{Z}_{2}\right)$ are completely determined by the fact that $S q^{1} d=c d$.

Proof of Theorem 1.1. Consider the projection map $q: D(m, n) \rightarrow \mathbb{R}^{m}$. Since the composition map,

$$
\mathbb{R P}^{m}=D(m, 0) \hookrightarrow D(m, n) \stackrel{q}{\rightarrow} \mathbb{R P}^{m},
$$

is the identity map, the induced map

$$
q^{*}: H^{i}\left(\mathbb{R P}^{m} ; \mathbb{Z}_{2}\right) \rightarrow H^{i}\left(D(m, n) ; \mathbb{Z}_{2}\right)
$$

is injective. Hence the suspension map induces an injective map

$$
\Sigma^{k} q^{*}: H^{i}\left(\Sigma^{k} \mathbb{R} \mathbb{P}^{m} ; \mathbb{Z}_{2}\right) \rightarrow H^{i}\left(\Sigma^{k} D(m, n) ; \mathbb{Z}_{2}\right) .
$$

Now if there is a vector bundle $\xi$ over $\Sigma^{k} \mathbb{R} \mathbb{P}^{m}$ with $w_{i}(\xi) \neq 0$, then $w_{i}\left(q^{*}(\xi)\right) \neq 0$. Thus we have showed that if $\Sigma^{k} \mathbb{R} \mathbb{P}^{m}$ is not $W$-trivial, then the $k$-fold suspension $\Sigma^{k} D(m, n)$ of Dold manifold $D(m, n)$ is also not $W$-trivial. Thus the proof of Theorem 1.1 now follows from Theorem 1.4 [11].

We now come to the proof of Theorem 1.2, which will be proved in sequence of the propositions below. 
Proposition 3.1. Let $m>0$. Then $\Sigma^{k} D(m, 2 r)$ is $W$-trivial if

1. $k=2$ and $m=1$.

2. $k=3$ and $m=8 t+2,8 t+3,8 t+4$ or $8 t+6$.

3. $k=5$ and $m=8 t, 8 t+4,8 t+5$ or $8 t+6$.

4. $k=6$ and $m=8 t+1,8 t+5,8 t+6$ or $8 t+7$.

5. $k=7$ and $m=8 t, 8 t+2,8 t+6$ or $8 t+7$.

Proof. Let $k$ and $m$ be as in the statement of the proposition. Note that the group $\widetilde{K O}^{-k}(m, 2 r)=0$ (Lemma 2.2) and the $k$-fold suspension $\Sigma^{k} \mathbb{R P}^{m}$ of $\mathbb{R P}^{m}$ is $W$-trivial ([11, Theorem 1.4]). Now by the decomposition,

$$
\widetilde{K O}^{-k}(D(m, 2 r))=\widetilde{K O}^{-k}(m, 2 r) \oplus q^{!} \widetilde{K O}^{-k}\left(\mathbb{R P}^{m}\right),
$$

of Theorem 2.1, any vector bundle $\xi \in \widetilde{K O}^{-k}(D(m, 2 r))$ is stably equivalent to $\eta \oplus \nu$, where $\eta \in \widetilde{K O}^{-k}(m, 2 r)$ and $\nu \in q^{!} \widetilde{K O}^{-k}\left(\mathbb{R P}^{m}\right)$. Since $W(\eta)=1$ and $W(\nu)=1$, we have $W(\xi)=1$. This completes the proof of the proposition.

Proposition 3.2. Let $m>0$. Then $\Sigma^{k} D(m, 2 r)$ is $W$-trivial if

1. $k=3$ and $m=8 t+5(t>0)$ or $8 t+7$.

2. $k=5$ and $m=8 t+1$ or $8 t+7$.

3. $k=6$ and $m=8 t$ or $8 t+2(t>0)$.

4. $k=7$ and $m=8 t+1(t>0)$ or $8 t+3$.

Proof. Let $k$ and $m$ be as in the statement of the proposition. Then consider the following exact sequence (2):

$$
\cdots \rightarrow \widetilde{K O}^{-k}\left(S^{m} \wedge \mathbb{C P}^{2 r}\right) \stackrel{f^{!}}{\rightarrow} \widetilde{K O}^{-k}(m, 2 r) \stackrel{i^{!}}{\rightarrow} \widetilde{K O}^{-k}(m-1,2 r) \rightarrow \cdots .
$$

Since $\widetilde{K O}^{-k}(m-1,2 r)=0$ (Lemma 2.2), the map $f^{!}$is surjective. By [11, Theorem 1.5], $\Sigma^{k+m} \mathbb{C P}^{2 r}$ is $W$-trivial, and this implies that for any vector bundle $\xi \in$ $\widetilde{K O}^{-k}(m, 2 r)$ the total Stiefel-Whitney class $W(\xi)=1$. Furthermore, we know that $\Sigma^{k} \mathbb{R} \mathbb{P}^{m}$ is $W$-trivial ([11, Theorem 1.4]). Hence by the decomposition,

$$
\widetilde{K O}^{-k}(D(m, 2 r))=\widetilde{K O}^{-k}(m, 2 r) \oplus q^{!} \widetilde{K O}^{-k}\left(\mathbb{R P}^{m}\right),
$$

of Theorem 2.1, we conclude that $\Sigma^{k} D(m, 2 r)$ is $W$-trivial.

Proposition 3.3. Let $m>0$. Then $\Sigma^{k} D(m, 2 r)$ is $W$-trivial if

1. $k=3$ and $m=8 t$.

2. $k=5$ and $m=8 t+2$.

3. $k=6$ and $m=8 t+3$.

4. $k=7$ and $m=8 t+4$.

5. $k=8$ and $m=1$. 
Proof. Let $k$ and $m$ be as in the statement of the proposition. Consider the inclusion

$$
i: D(m-1,2 r) \hookrightarrow D(m, 2 r) .
$$

Note that the quotient

$$
D(m, 2 r) / D(m-1,2 r) \approx \Sigma^{m} \mathbb{C P}^{2 r} \vee S^{m},
$$

and hence the induced map

$$
i^{*}: H^{p}\left(D(m, 2 r) ; \mathbb{Z}_{2}\right) \rightarrow H^{p}\left(D(m-1,2 r) ; \mathbb{Z}_{2}\right)
$$

is a monomorphism when $p+m$ is odd. Thus the induced suspension map

$$
\Sigma^{k} i^{*}: H^{q}\left(\Sigma^{k} D(m, 2 r) ; \mathbb{Z}_{2}\right) \rightarrow H^{q}\left(\Sigma^{k} D(m-1,2 r) ; \mathbb{Z}_{2}\right)
$$

is a monomorphism for even $q$.

Now, if there is a vector bundle $\xi$ over $\Sigma^{k} D(m, 2 r)$ with $w_{2^{s}}(\xi) \neq 0$ for some $s>0$, then $w_{2^{s}}\left(i^{*}(\xi)\right) \neq 0$. But we know that $\Sigma^{k} D(m-1,2 r)$ is $W$-trivial (refer to Proposition 3.2 for $k \neq 8$ and [11, Theorem 1.5] for $k=8$ ). This gives a contradiction, and thus we conclude that $\Sigma^{k} D(m, 2 r)$ is $W$-trivial.

Proposition 3.4. The iterated suspensions $\Sigma^{8} D(2,2 r)$ and $\Sigma^{8} D(3,2 r)$ are $W$-trivial.

Proof. We shall first prove that $\Sigma^{8} D(2,2 r)$ is $W$-trivial. Let $\xi$ be a vector bundle over $\Sigma^{8} D(2,2 r)$. Let $s \geqslant 4$ be such that $2^{s} \leqslant \operatorname{dim}\left(\Sigma^{8} D(2,2 r)\right)$ and $w_{j}(\xi)=0$ for $0<$ $j<2^{s}$. We shall show that $w_{2^{s}}(\xi)=0$, and thus this will imply that $\Sigma^{8} D(2,2 r)$ is $W$-trivial.

Let $a \in H^{2^{s}-8}\left(D(2,2 r) ; \mathbb{Z}_{2}\right)$ be the cohomology class that maps to

$$
w_{2^{s}}(\xi) \in H^{2^{s}}\left(\Sigma^{8} D(2,2 r) ; \mathbb{Z}_{2}\right)
$$

under the suspension isomorphism

$$
H^{2^{s}-8}\left(D(2,2 r) ; \mathbb{Z}_{2}\right) \rightarrow H^{2^{s}}\left(\Sigma^{8} D(2,2 r) ; \mathbb{Z}_{2}\right)
$$

By $[\mathbf{1 1}$, Lemma 3.3] and the fact that the Steenrod squares commutes with suspension homomorphism, we have

$$
S q^{i}(a)=0 \text { for all } 0<i<2^{s-1} .
$$

Now observe that the vector space $H^{2^{s}-8}\left(D(2,2 r) ; \mathbb{Z}_{2}\right)$ is generated by

$$
d^{2^{s-1}-4} \text { and } c^{2} d^{2^{s-1}-5} \text {. }
$$

Therefore,

$$
a=x \cdot d^{2^{s-1}-4}+y \cdot c^{2} d^{2^{s-1}-5}
$$

for $x, y \in \mathbb{Z}_{2}$. If $x \neq 0$ then $w_{2^{s}}\left(i^{*} \xi\right) \neq 0$, where

$$
i: \Sigma^{8} \mathbb{C P}^{2 r} \hookrightarrow \Sigma^{8} D(2,2 r)
$$

is the inclusion map and $i^{*} \xi$ is the pullback bundle over $\Sigma^{8} \mathbb{C P}^{2 r}$. But since $\Sigma^{8} \mathbb{C P}^{2 r}$ is $W$-trivial ([11, Theorem 1.5]), we have a contradiction and hence $x=0$. Further, 
since

$$
S q^{2}\left(c^{2} d^{2^{s-1}-5}\right)=c^{2} d^{2^{s-1}-4} \neq 0,
$$

we have $y=0$. Hence,

$$
w_{2^{s}}(\xi)=0 .
$$

This completes the proof of $W$-triviality of $\Sigma^{8} D(2,2 r)$.

The proof of $W$-triviality of $\Sigma^{8} D(3,2 r)$ proceeds along the same lines as the proof of Proposition 3.3 using the fact that $\Sigma^{8} D(2,2 r)$ is $W$-trivial.

In the following proposition, $n$ can be both even or odd:

Proposition 3.5. The iterated suspensions $\Sigma^{4} D(3, n)$ and $\Sigma^{8} D(7, n)$ are $W$-trivial.

Proof. Let $s \geqslant 3$ be such that $2^{s} \leqslant \operatorname{dim}\left(\Sigma^{4} D(3, n)\right)$. Observe that the vector space $H^{2^{s}-4}\left(D(3, n) ; \mathbb{Z}_{2}\right)$ is generated by

$$
d^{2^{s-1}-2} \text { and } c^{2} d^{2^{s-1}-3} .
$$

Here note that $d^{2^{s-1}-2}$ will be zero if $n<2^{s-1}-2$. Further observe that

$$
S q^{1}\left(c^{2} d^{2^{s-1}-3}\right)=c^{3} d^{2^{s-1}-3} \neq 0 .
$$

With these observations the proof of the $W$-triviality of $\Sigma^{4} D(3, n)$ proceeds along the same lines as the proof of $W$-triviality of $\Sigma^{8} D(2,2 r)$ in Proposition 3.4.

Similarly we can argue that $\Sigma^{8} D(7, n)$ is $W$-trivial. Here we need to observe that, for $s \geqslant 4$ such that $2^{s} \leqslant \operatorname{dim}\left(\Sigma^{8} D(7, n)\right)$, the vector space $H^{2^{s}-8}\left(P(7, n) ; \mathbb{Z}_{2}\right)$ is generated by

$$
d^{2^{s-1}-4}, c^{2} d^{2^{s-1}-5}, c^{4} d^{2^{s-1}-6} \text { and } c^{6} d^{2^{s-1}-7} .
$$

Here again some of these cohomology classes, except $c^{6} d^{2^{s-1}-7}$, can be zero. Further observe that

$$
S q^{1}\left(c^{2} d^{2^{s-1}-5}\right)=c^{3} d^{2^{s-1}-5}, S q^{1}\left(c^{6} d^{2^{s-1}-7}\right)=c^{7} d^{2^{s-1}-7} \text { and } S q^{1}\left(c^{4} d^{2^{s-1}-6}\right)=0,
$$

but $S q^{2}\left(c^{4} d^{2^{s-1}-6}\right)=c^{6} d^{2^{s-1}-6}$. Now the proof of $W$-triviality of $\Sigma^{8} D(7, n)$ will proceed along the same lines as in the case $\Sigma^{4} D(3, n)$. This completes the proof of the proposition.

Remark 3.6. More generally, one can prove that the $m$-fold suspension $\Sigma^{m} D(m-$ $1, n)$ of the Dold manifold $D(m-1, n)$ with $m>0$ is $W$-trivial by the method used in proving Proposition 3.5.

The proof of Theorem 1.2 follows from Propositions 3.1, 3.2, 3.3, 3.4 and 3.5.

We now come to the proof of Theorem 1.3. First we make the following observation concerning the $W$-triviality of stunted projective space.

Lemma 3.7. Let $\mathbb{R} \mathbb{P}^{m} / \mathbb{R} \mathbb{P}^{n}$ be the stunted projective space with $m \geqslant n$. Then $\Sigma^{k}\left(\mathbb{R P}^{m} / \mathbb{R P}^{n}\right)$ is $W$-trivial if

1. $k=1,2,4$ or 8 and $m<k$.

2. $k=3,5$ or 7 and $m+k \neq 4,8$.

3. $k=6$ and $m \neq 2,3$.

4. $k \geqslant 9$. 
Proof. Let $X=\mathbb{R} \mathbb{P}^{m} / \mathbb{R} \mathbb{P}^{n}$, and let $\alpha: \mathbb{R} \mathbb{P}^{m} \rightarrow X$ be the projection map. Then the induced suspension homomorphism

$$
\left(\Sigma^{k} \alpha\right)^{*}: H^{i}\left(\Sigma^{k} X ; \mathbb{Z}_{2}\right) \rightarrow H^{i}\left(\Sigma^{k} \mathbb{R} \mathbb{P}^{m} ; \mathbb{Z}_{2}\right)
$$

is an isomorphism for $i>n+k$. Hence if there is a vector bundle $\xi$ over $\Sigma^{k} X$ with $w_{i}(\xi) \neq 0$, then $w_{i}\left(\left(\Sigma^{k} \alpha\right)^{*} \xi\right) \neq 0$. Thus the $W$-triviality of $\Sigma^{k} \mathbb{R} \mathbb{P}^{m}$ implies the $W$ triviality of $\Sigma^{k} X$. Now the proof of the lemma follows from [11, Theorem 1.4].

Proposition 3.8. Let $\Sigma^{k} D(m, 2 r+1)$ be the $k$-fold suspension of the Dold manifold $D(m, 2 r+1)$. Then $\Sigma^{k} D(m, 2 r+1)$ is $W$-trivial if

$$
\Sigma^{k} D(m, 2 r+2) \text { and } \Sigma^{2 r+1+k}\left(\mathbb{R P}^{2 r+1+m} / \mathbb{R P}^{2 r}\right)
$$

are $W$-trivial.

Proof. Let $\xi$ be a vector bundle over $\Sigma^{k} D(m, 2 r+1)$. We shall prove the proposition by showing that the total Stiefel-Whitney class $W(\xi)=1$.

Consider the following decomposition by Theorem 2.1:

$$
\widetilde{K O}^{-k}(D(m, 2 r+1))=\widetilde{K O}^{-k}(m, 2 r+1) \oplus \widetilde{K O}^{-k}\left(\mathbb{R P}^{m}\right) .
$$

Since the $W$-triviality of $\Sigma^{k} D(m, 2 r+2)$ implies the $W$-triviality of $\Sigma^{k} \mathbb{R} \mathbb{P}^{m}$ (Theorem 2.1), we can assume that $\xi \in \widetilde{K O}^{-k}(m, 2 r+1)$. Now, since the $(2 r+1+k)$-fold suspension $\Sigma^{2 r+1+k}\left(\mathbb{R P}^{2 r+1+m} / \mathbb{R P}^{2 r}\right)$ is $W$-trivial and by the decomposition,

$$
\widetilde{K O}^{-k}(m, 2 r+1)=\widetilde{K O}^{-k}(m, 2 r) \oplus \widetilde{K O}^{-k}\left(\Sigma^{2 r+1}\left(\mathbb{R P}^{2 r+1+m} / \mathbb{R P}^{2 r}\right)\right),
$$

of Theorem 2.3, we can further assume that $\xi=\kappa(\gamma)$ for some $\gamma \in \widetilde{K O}^{-k}(m, 2 r)$. Here $\kappa$ is the monomorphism with respect to which $\widetilde{K O}^{-k}(m, 2 r)$ is a direct summand of $\widetilde{K O}^{-k}(m, 2 r+1)$ (refer to $[\mathbf{6}$, Section 10] for more details). By the definition of $\kappa$, we have $\xi=i_{2}^{!}(\eta)$ for some $\eta \in \widetilde{K O}^{-k}(m, 2 r+2)$. Here $i_{2}: D(m, 2 r+1) \hookrightarrow$ $D(m, 2 r+2)$ is the inclusion. Thus, by the $W$-triviality of $\Sigma^{k} D(m, 2 r+2)$, we conclude that $W(\xi)=1$. This completes the proof of the proposition.

Now, under the given hypothesis on $k, n=2 r+1$ and $m$, as in statement of Theorem 1.3, the $k$-fold suspension $\Sigma^{k} D(m, 2 r+2)$ is $W$-trivial for all $r \geqslant 0$ (Theorem 1.2). It is also clear by Lemma 3.7 that for these values of $k, n=2 r+1$ and $m$, the space $\Sigma^{2 r+1+k}\left(\mathbb{R} \mathbb{P}^{2 r+1+m} / \mathbb{R P}^{2 r}\right)$ is $W$-trivial. Thus the proof of Theorem 1.3 follows from Proposition 3.8.

We now come to the proof of Theorem 1.4. First note the following lemma:

Lemma 3.9. The $k$-fold suspension $\Sigma^{k} D(m, n)$ is not $W$-trivial if the $(n+k)$-fold suspension $\Sigma^{n+k}\left(\mathbb{R} \mathbb{P}^{n+m} / \mathbb{R} \mathbb{P}^{n-1}\right)$ is not $W$-trivial.

Proof. Observe that the inclusion $i: D(m, n-1) \hookrightarrow D(m, n)$ induces a surjective map 


$$
i^{*}: H^{p}\left(D(m, n) ; \mathbb{Z}_{2}\right) \rightarrow H^{p}\left(D(m, n-1) ; \mathbb{Z}_{2}\right), \text { for all } p
$$

in cohomology group. Thus

$$
\pi^{*}: H^{p}\left(D(m, n) / D(m, n-1) ; \mathbb{Z}_{2}\right) \rightarrow H^{p}\left(D(m, n) ; \mathbb{Z}_{2}\right), \text { for } p>0
$$

is injective. Here

$$
\pi: D(m, n) \rightarrow D(m, n) / D(m, n-1) \approx \Sigma^{n}\left(\mathbb{R P}^{n+m} / \mathbb{R} \mathbb{P}^{n-1}\right)
$$

is the quotient map. Thus the induced suspension morphism

$$
\Sigma^{k} \pi^{*}: H^{p}\left(\Sigma^{n+k}\left(\mathbb{R} \mathbb{P}^{n+m} / \mathbb{R} \mathbb{P}^{n-1}\right) ; \mathbb{Z}_{2}\right) \rightarrow H^{p}\left(\Sigma^{k} D(m, n) ; \mathbb{Z}_{2}\right)
$$

is injective for $p>0$. Hence if there is a vector bundle $\xi$ over $\Sigma^{n+k}\left(\mathbb{R} \mathbb{P}^{n+m} / \mathbb{R} \mathbb{P}^{n-1}\right)$ with $W(\xi) \neq 1$, then $W\left(\pi^{*}(\xi)\right) \neq 1$. This completes the proof of the lemma.

Now the proof of Theorem 1.4 follows immediately from Lemma 3.9 and [11, Theorem 1.4].

This completes the proof of the results stated in the introduction. As noted earlier we still do not know whether $\Sigma^{3} D(m, 5)$ and $\Sigma^{5} D(m, 3)$ are $W$-trivial or not for all $m$. Though this can be answered in few cases, we do not have a complete picture. For example, if $m=2,3$ or 4 then $\Sigma^{3} D(m, 5)$ is $W$-trivial by Proposition 3.8 and [11, Corollary 1.3]. By a similar argument we can say that if $m=1,2,4,5$ or 6 , then $\Sigma^{5} D(m, 3)$ is $W$-trivial. We also have the following proposition:

Proposition 3.10. Let $n>1$ and $n \not \equiv 3(\bmod 4)$. Then $\Sigma^{4} D(1, n)$ is not $W$-trivial.

Proof. Consider the following long exact sequence (2):

$$
\cdots \rightarrow \widetilde{K O}^{-4}\left(\Sigma \mathbb{C P}^{n}\right) \stackrel{f^{!}}{\rightarrow} \widetilde{K O}^{-4}(1, n) \stackrel{i^{!}}{\rightarrow} \widetilde{K O}^{-4}(0, n) \stackrel{\delta}{\rightarrow} \widetilde{K O}^{-3}\left(\Sigma \mathbb{C P}^{n}\right) \rightarrow \cdots .
$$

Since $\widetilde{K O}^{-4}\left(\Sigma \mathbb{C P}^{n}\right)=0[\mathbf{3}$, Theorem 2], the homomorphism

$$
i^{!}: \widetilde{K O}^{-4}(1, n) \longrightarrow \widetilde{K O}^{-4}(0, n)
$$

is a monomorphism. We shall first prove that $i$ is an isomorphism.

Depending upon whether $n$ is odd or even, we write $n=2 r$ or $2 r+1$. As $n \not \equiv 3$ ( $\bmod 4)$ we have, by $[\mathbf{3}$, Theorem 2],

$$
\widetilde{K O}^{-4}\left(\mathbb{C P}^{n}\right)=\widetilde{K O}^{-4}(0, n)=\mathbb{Z}^{r}
$$

Now if $n=2 r+1$, then we have the decomposition

$$
\widetilde{K O}^{-4}(1,2 r+1)=\widetilde{K O}^{-4}(1,2 r) \oplus \widetilde{K O}^{-4}\left(\Sigma^{2 r+1}\left(\mathbb{R P}^{2 r+2} / \mathbb{R P}^{2 r}\right)\right)
$$

by Theorem 2.3. We have $\widetilde{K O}^{-4}\left(\Sigma^{2 r+1}\left(\mathbb{R P}^{2 r+2} / \mathbb{R P}^{2 r}\right)\right)=0$ (refer to Table (2) on $\left[\mathbf{5}\right.$, p. 47]) and $\widetilde{K O}^{-4}(1,2 r)=\mathbb{Z}^{r}[\mathbf{6}$, Theorem 3]. Therefore,

$$
\widetilde{K O}^{-4}(1, n)=\mathbb{Z}^{r} \text {. }
$$

Now putting all these values of $\widetilde{K O}$-groups in the above long exact sequence one can easily conclude that the homomorphism

$$
\delta: \widetilde{K O}^{-4}(0, n) \longrightarrow \widetilde{K O}^{-3}\left(\Sigma \mathbb{C P}^{n}\right)=\widetilde{K O}^{-4}\left(\mathbb{C P}^{n}\right)
$$

is zero. Thus the homomorphism $i^{!}$is an isomorphism. 
As $\Sigma^{4} \mathbb{C} \mathbb{P}^{n}$ is not $W$-trivial $([\mathbf{1 1}$, Theorem 1.5$])$, there is a vector bundle $\xi \in$ $\widetilde{K O}^{-4}(0, n)=\widetilde{K O}^{-4}\left(\mathbb{C P}^{n}\right)$ with

$$
W(\xi) \neq 1
$$

Thus there is a vector bundle over $\Sigma^{4} D(1, n)$ with non-trivial Stiefel-Whitney class. This completes the proof of the proposition.

Remark 3.11. Note that there is no integer $s$ such that $5 \leqslant 2^{s} \leqslant \operatorname{dim}\left(\Sigma^{4} D(1,1)\right)=7$. Hence $\Sigma^{4} D(1,1)$ is $W$-trivial.

\section{References}

[1] M.F. Atiyah and F. Hirzebruch, Bott periodicity and the parallelizability of the spheres, Proc. Cambridge Philos. Soc. 57 (1961), no. 2, 223-226.

[2] A. Dold, Erzeugende der Thomschen Algebra N, Math. Z. 65 (1956), no. 1, $25-35$.

[3] M. Fujii, $K_{O}$-groups of projective spaces, Osaka J. Math. 4 (1967), no. 1, 141149.

[4] M. Fujii, Ring structures of $K_{U}$-cohomologies of Dold manifolds, Osaka J. Math. 6 (1969), no. 1, 107-115.

[5] M. Fujii and T. Yasui, $K_{O}$-groups of the stunted real projective spaces, Math. J. Okayama Univ. 16 (1973), no. 1, 47-54.

[6] M. Fujii and T. Yasui, $K_{O}$-cohomologies of the Dold manifolds, Math. J. Okayama Univ. 16 (1973), no. 1, 55-84.

[7] J. Milnor, Some consequences of a theorem of Bott, Ann. of Math. 68 (1958), no. 2, 444-449.

[8] J. Milnor and J.D. Stasheff, Characteristic classes, Annals of Mathematics Studies 76, Princeton University Press, Princeton, NJ, 1974.

[9] R. Tanaka, On the index and co-index of sphere bundle, Kyushu J. Math. 57 (2003), no. 2, 371-382.

[10] R. Tanaka, On trivialities of Stiefel-Whitney classes of vector bundles over highly connected complexes, Topology Appl. 155 (2008), no. 15, 1687-1693.

[11] R. Tanaka, On trivialities of Stiefel-Whitney classes of vector bundles over iterated suspension spaces, Homology, Homotopy Appl. 12 (2010), no. 1, 357366.

[12] R. Tanaka, A Borsuk-Ulam type theorem over iterated suspensions of real projective spaces, J. Korean Math. Soc. 49 (2012), no. 2, 251-263.

Ajay Singh Thakur athakur@math.tifr.res.in

School of Mathematics, Tata Institute of Fundamental Research, Homi Bhabha Road, Colaba, Mumbai 400 005, India 